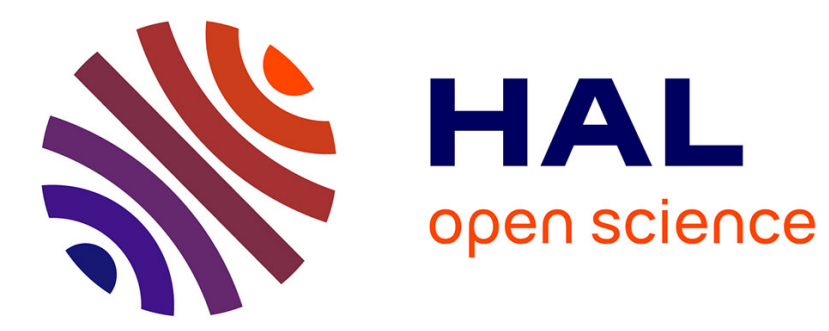

\title{
Paraxial imaging and walk-off effects with birefringent media in refracting surfaces, lenses, and slabs
}

\author{
Luc Dettwiller
}

\section{To cite this version:}

Luc Dettwiller. Paraxial imaging and walk-off effects with birefringent media in refracting surfaces, lenses, and slabs. Applied optics, 2008, 47 (3), pp.442-452. 10.1364/AO.47.000442 . hal-00880188

\section{HAL Id: hal-00880188 \\ https://hal-iogs.archives-ouvertes.fr/hal-00880188}

Submitted on 5 Nov 2013

HAL is a multi-disciplinary open access archive for the deposit and dissemination of scientific research documents, whether they are published or not. The documents may come from teaching and research institutions in France or abroad, or from public or private research centers.
L'archive ouverte pluridisciplinaire HAL, est destinée au dépôt et à la diffusion de documents scientifiques de niveau recherche, publiés ou non, émanant des établissements d'enseignement et de recherche français ou étrangers, des laboratoires publics ou privés. 


\title{
Paraxial imaging and walk-off effects with birefringent media in refracting surfaces, lenses, and slabs
}

\author{
Luc Dettwiller ${ }^{1,2}$ \\ ${ }^{1}$ Lycée Blaise Pascal, 36 avenue Carnot, F63037 Clermont-Ferrand Cedex, France \\ 2Laboratoire Charles Fabry de l'Institut d'Optique, CNRS, Campus polytechnique, Université Paris Sud, 2 avenue Fresnel, \\ F91127 Palaiseau Cedex, France \\ detwiller.luc@voila.fr
}

Received 2 July 2007; revised 14 November 2007; accepted 18 November 2007; posted 11 December 2007 (Doc. ID 84721); published 18 January 2008

\begin{abstract}
The paraxial theory of spherical refracting surfaces, spherical lenses, and slabs with one birefringent medium is investigated analytically: using walk-off effects in the paraxial domain, a number of relations between objects and images are deduced, along with cardinal elements, in the case where the optic axis is parallel to the optical axis. This method naturally shows that in some cases first-order astigmatism appears. An argument based on the wavefront (and phase) transformation shows that any spherical birefringent thin lens is stigmatic in the paraxial domain, because the first-order astigmatisms due to the two surfaces of such a lens compensate each other. This is a priori not the case with thick birefringent lenses-but two such cases are detailed. () 2008 Optical Society of America

OCIS codes: $\quad 080.0080,080.2730,260.1180,260.1440$.
\end{abstract}

\section{Introduction}

The theory of some birefringent lenses has been recently addressed [1-5]. Some works, such as [4], mention that such lenses are used in various systems: common path profilometers [6,7], compact-disk readers [8], image processors [9], intraocular prosthesis [10], etc. Even in the case of a uniaxial crystal whose optic axis is parallel or perpendicular to the geometrical axis of the lens, the problem with a birefringent medium arises with the refraction law, for which various expressions have been given [11-24] in the past decades, often using Huygens's construction or eventually Fermat's principle-which both yield the refracted rays directly. With Descartes's construction, for a ray propagating from an isotropic to an anisotropic medium, there are two steps (see the caption of Fig. 1, which explains an example of construction): the first one gives the two refracted wave normals (see, for example, [25,26]); the second gives the two refracted rays, which generally are not parallel to their normals. The splitting, due to the medium an-

0003-6935/08/030442-11\$15.00/0

(C) 2008 Optical Society of America isotropy, between a wave normal and-at least-one of the corresponding rays, is called walk-off phenomenon. Several methods to find the directions of these rays are available, and here it will be interesting to proceed in a slightly different way than the latter references do: we can use the well-known walk-off property ([27], pp. 462-463) that each of these two rays is perpendicular to the respective sheet of the index surface, at the point corresponding to its wave normal-if the birefringent medium is local [28] (i.e., concerning a dielectric, if the electric displacement at any point $P$ of the medium depends on the electric field at $P$ only).

In this paper, my goal is to give a deeper understanding of paraxial imaging with a birefringent system: all the incident rays considered are near the line $\left(\Delta_{g}\right)$ - called "optical axis" of the system. First, I will discuss the effect of the walk-off on the refraction for small incidences, and I will introduce an apparent refractive index that is a curvature radius of a section of the index surface. Then, I will deduce the optical properties of an elementary system such as a plane or spherical [29] refracting surface between an isotropic and an anisotropic medium; and I will show that when, for example, the latter is uniaxial and its optic 
axis parallel to $\left(\Delta_{g}\right)$ normal to the surface, cardinal points can be defined even for the extraordinary beams. As a consequence, a number of relations between objects and images are the same as for a usual spherical refracting surface-but its nodal point is no more its center. Finally, I will apply these results to the study of lenses, and stress a remarkable simplifying fact: for a thin lens, the walk-off effects on the front and the back side of the lens cancel each other, even with a biaxial medium; but this is no more valid for a slab or a thick lens, which may be fundamentally astigmatic, i.e., show a first-order astigmatism, hence even for an object point on $\left(\Delta_{g}\right)$, such as a cylindrical isotropic lens-if the optic axis is not parallel to $\left(\Delta_{g}\right)$ [3-5]. Incidentally, I will indicate that for some uniaxial slabs the extraordinary paraxial images always coincide with the objects.

\section{Kepler's Law Generalized to an Anisotropic Medium}

Kepler's law is a first-order approximation of Snell's refraction law; the former is sufficient for the theory of paraxial imaging. Let us recall that the refraction law dealing with the wave normals is like the classical Snell's refraction law, except that for an anisotropic medium there are two refractive indices, one of them at least depending on the direction of the wave normal. But for the imaging theory we have to determine the directions of the refracted rays, which implies taking the walk-off phenomenon into account. For the remainder of this paper, the refractive indices used will be relative to the input (or object space) medium, assumed to be isotropic.

\section{A. Introductory Example}

Let us consider the case when the output medium is uniaxial. The angle $\alpha$ between its optic axis $\left(\Delta_{o}\right)$ and the normal $\left(\mathcal{N}_{O}\right)$ to the refracting surface $(D)$ at the incidence point $O$ is assumed to be of first order, as are the angles of incidence and refraction in Kepler's law (see Fig. 1); let us call $n_{o}$ and $n_{e}$ its ordinary and extraordinary (relative) refractive indices.

Consider an incident ray located in the principal section plane $\left(\Pi_{O}\right)$ at $O$, i.e., the plane parallel to $\left(\Delta_{o}\right)$ and containing $\left(\mathcal{N}_{O}\right)$; in this particular simple casedue to the symmetry of the incident ray, of each sheet of the index surface, and of $\left(\mathcal{N}_{O}\right)$, with respect to $\left(\Pi_{O}\right)$ - each refracted ray lies in $\left(\Pi_{O}\right)$. Because the ordinary ray behaves as if the medium was isotropic (with refractive index $n_{o}$ ), in this subsection we study only the case of the extraordinary rays. Let us call $\theta$ and $\theta^{\prime}$ the incidence and refraction angles. The rays being normal to the index surface used for Descartes's construction, their directions depend on the curvature of this surface.

First, the extraordinary ray is perpendicular to the extraordinary sheet $\left(\Sigma_{e}\right)$ of the index surface at the point $K$ for $\theta=0$ and $\alpha \neq 0$ (see Fig. 1), but at the point $U$ on $\left(\Delta_{o}\right)$ for $\theta=\alpha=0$. Let us recall that $\left(\Sigma_{o}\right)$, the ordinary sheet of the index surface, and $\left(\Sigma_{e}\right)$ are, respectively, a sphere (of radius $n_{o}$ ) and an ellipsoid, of center $O$ and revolution axis $\left(\Delta_{o}\right)$. Then the firstorder approximations (versus $\alpha$ ) of the algebraic

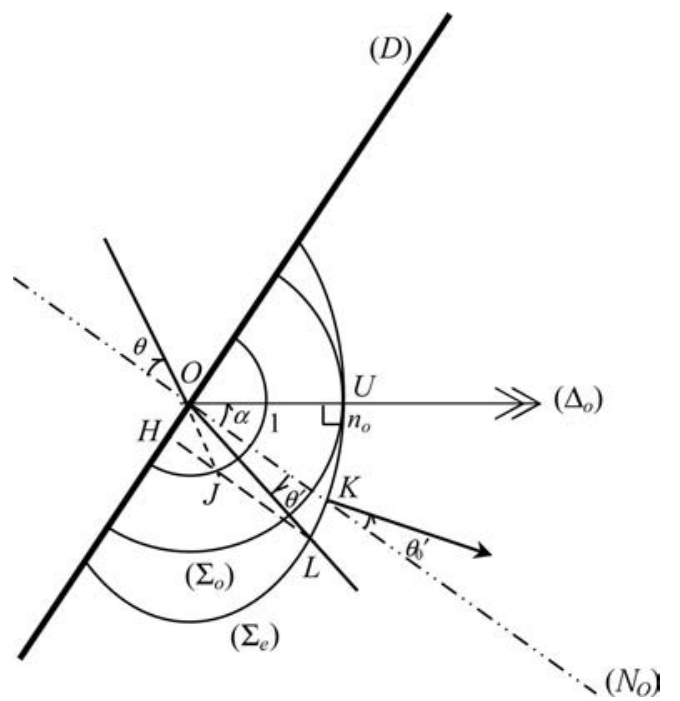

Fig. 1. Explanation of Kepler's law (hence for small $\theta$ ) by Descartes's construction with a uniaxial crystal and small $\alpha$. Note that what is called $(D)$ in Figs. 1 and 2 may be the plane tangent to the refracting surface at the incidence point, if this surface is curved. Descartes's construction consists in drawing, for the incident ray at $O$, its continuation (line with small dashes), which intersects the input index surface at $J$; next to consider $H J$ (dashed line), perpendicular to $(D)$ and intersecting $\left(\Sigma_{e}\right)$ at $L$ : the direction of the extraordinary refracted wave normal [ray] is parallel to $O L$ [perpendicular to $\left(\Sigma_{e}\right)$ at $L$, as is for $\theta=0$ (the arrow beginning at $K)]$.

length $\widehat{l}_{0}$ of the small plane arc $U K$ and of the refraction angle of the ray for $\theta=0$ are, respectively,

$$
\widehat{l}_{0} \cong-n_{o} \alpha, \quad \theta_{0}{ }^{\prime} \cong\left(n_{A}{ }^{-1}-n_{o}{ }^{-1}\right) \widehat{l}_{0},
$$

because with $\theta=0$ and $\left(\Delta_{o}\right)$ fixed but $\alpha$ varying near zero, from $U$ to $K$ the first-order angular variation of the orientation of the perpendicular to $\left(\Sigma_{e}\right)$ resp. $\left(\Sigma_{o}\right)$ is $\bar{l}_{0} / n_{A}$ resp. $\left(\bar{l}_{0} / n_{o}\right)$ where $n_{A}=n_{e}{ }^{2} / n_{o}$ is the radius of curvature of $\left(\Sigma_{e}\right)$ at $U$ : due to the rotational symmetry of $\left(\Sigma_{e}\right)$ around $\left(\Delta_{o}\right)$, the point $U$ is an umbilic (i.e., its principal curvatures are equal) [30]. Here, the important property of $n_{A}$ is to give easily the first-order angular variation of the orientation of the perpendicular to $\left(\Sigma_{e}\right)$; like $n_{o}$ for the sphere $\left(\Sigma_{o}\right)$.

For $\theta \neq 0$, the Descartes's construction shows that the refracted ray is normal to $\left(\Sigma_{e}\right)$ at $L$ (see Fig. 1), and the first-order approximation in $\theta$ and in $\alpha$ gives the algebraic length $\widehat{l}$ of the small plane arc $K L$ :

$$
\widehat{l} \cong \overline{O H} \cong O J \times \theta=\theta, \quad \theta^{\prime} \cong \theta_{0}{ }^{\prime}+\frac{\widehat{l}}{n_{A}} .
$$

In this particular case, Kepler's law is

$$
n_{A} \theta^{\prime} \cong \theta+\left(n_{A}-n_{o}\right) \alpha
$$

(if the output medium is isotropic this reduces evidently to the ordinary Kepler's law, because $n_{A}=n_{o}=n_{e} \equiv n ; \alpha$ is no more relevant). We can check our Eq. (3): it is a total first-order expansion (in $\theta$ and 


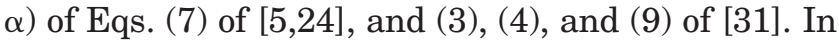
some cases of refraction indeed (see, for example, Subsections 3.A, 3.C, and 4.A.1) the radius of curvature $n_{A}$ of a section of the index surface is very useful, because it behaves like an apparent refractive index. It is never between $n_{o}$ and $n_{e}: n_{A}>n_{e}$ for a positive uniaxial medium (e.g., quartz) because $n_{e}>n_{o}$, which corresponds to the case represented in Fig. 1; for a negative medium (e.g., calcite) $n_{A}<n_{e}$ because $n_{e}<n_{o}$. In both cases the derivation leading to Eqs. (1)-(3) is valid, thanks to the algebraic nature of the lengths and the angles involved.

\section{B. More General Case}

In more complicated cases (for example, when the incident ray is not in the principal section plane, or $\alpha$ is not small, or the output medium is biaxial), a vectorial analysis of the Descartes's construction is useful. As we will see, it leads to a simple first-order expression for the variation $\delta \mathbf{r}^{\prime}$, with respect to the normal incidence case when $O$ is kept constant, of the unit vector $\mathbf{r}^{\prime}$ parallel to the refracted ray.

For the case of a normally incident ray in an isotropic input medium, consider one of the two refracted rays (directed by $\mathbf{r}_{N}{ }^{\prime}$ ) in the output anisotropic medium. It is related with the wave vector $k \mathbf{O M}$ in this medium (with $k=2 \pi / \lambda$ where $\lambda$ denotes the wavelength in a vacuum); $M$ is the point, determined by the Descartes's construction, of the sheet $\left(\Sigma^{\prime}\right)$ of the anisotropic index surface of center $O$ [see Fig. 2(a)].

Now let us examine the effect of small tilts of $\left(\mathcal{N}_{O}\right)$ and of the incident ray, when the incidence point $O$ and the crystallographic axes of the output medium-hence also its index surface-remain fixed [see Fig. 2(b)]: this problem prepares the study of a refracted pencil of rays from an incident pencil on a curved surface. In the output medium the small variation, with respect to $k \mathbf{O M}$, of the wave vector is $k \mathbf{M Q}$, where $Q$ is determined by Descartes's construction [see Fig. 2(b)]. The transverse (i.e., normal to OM) component of the global displacement $\mathbf{M Q}$ is the result of independent contributions combining additively always in the first-order approximation.

- $\left(n_{N}-1\right) \delta \mathbf{v}$, due to the small change $\delta \mathbf{v}$ of the unit normal $\mathbf{v}$ to $(D)$ at $O$ (turned from the input to the output medium), $n_{N}=\|\mathbf{O M}\|$ being the abscissa of $M$, hence the refractive index associated to the refracted wave normal considered in the case of the normal incidence on $(D)$ initially not tilted [case of the incident horizontal ray on Fig. 2(b); see also its caption]; in Descartes's construction for this incident horizontal ray, the tilt of only $(D)$ rotates the dashed perpendicular to $(D)$ around the point marked by its abscissa 1 [see Fig. 2(b)] and distant from $M$ by $n_{N}-1$

- $\delta \mathbf{r}$, due to the small change of the unit vector $\mathbf{r}$ oriented like the incident ray. Let us call $(\mathbf{P})$ the $2 \times 2$ matrix of the orthogonal projection from the plane $\left(\pi^{\prime}\right)$, tangent to $\left(\Sigma^{\prime}\right)$ at $M$, onto the plane normal to $\mathbf{O M}$. Because these planes are not normal, $(\mathbf{P})^{-1}$ exists and

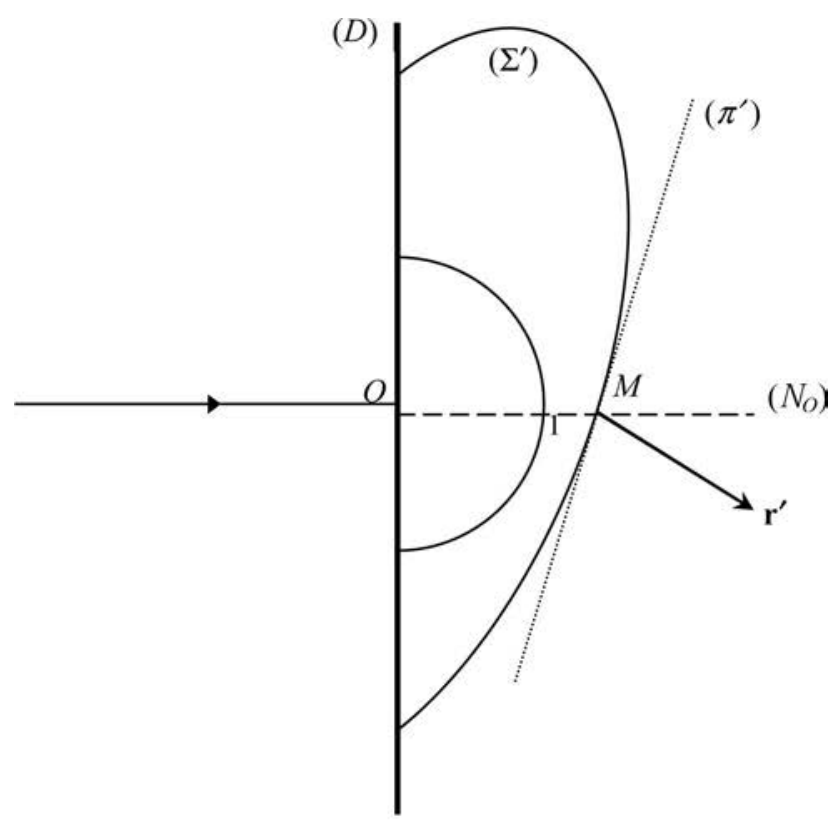

(a)

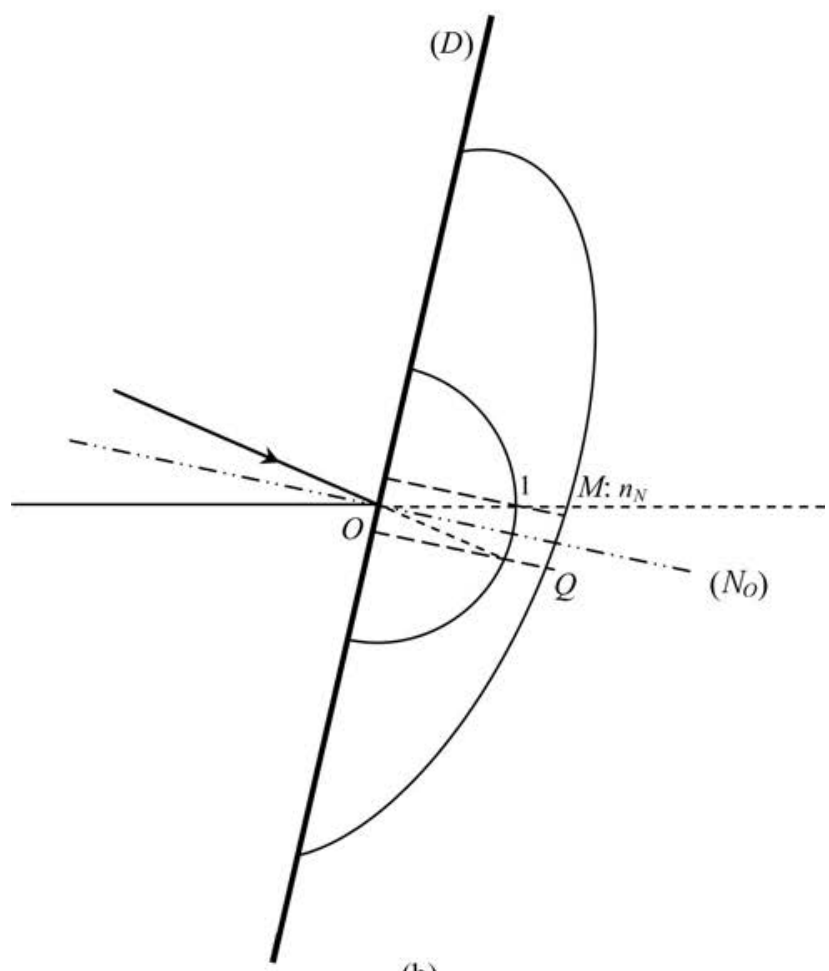

(b)

Fig. 2. Vectorial analysis of the Descartes's construction. (a) Case of a normally incident ray. (b) Effect of small tilts of $\left(\mathcal{N}_{O}\right)$ and of the incident ray. The index surfaces remain fixed, and the upper dashed line normal to $(D)$ shows Descartes's construction when the incident ray also remains fixed (horizontal on this figure) and only $\left\{(D),\left(\mathcal{N}_{O}\right)\right\}$ is slightly tilted, leading to the term in $\delta \mathbf{v}$; the translation from the upper to the lower dashed line is due to $\delta \mathbf{r}$, and shows the change in Descartes's construction when the incident ray is slightly rotated.

$$
\mathbf{M Q} \cong(\mathbf{P})^{-1}\left[\left(n_{N}-1\right) \delta \mathbf{v}+\delta \mathbf{r}\right] .
$$

Equation (4) leads to the first-order approximation of the variation $k \mathbf{M Q}$ of the wave vector $k n^{\prime} \mathbf{u}^{\prime}$ (where $\mathbf{u}^{\prime}$ 
is the wave normal associated in the medium to the refractive index $n^{\prime}$ and to the refracted ray considered); we now have to express the variation $\delta \mathbf{r}^{\prime}$ of the unit vector $\mathbf{r}^{\prime}$ oriented like this refracted ray in the anisotropic output medium-eventually nonlocal (see Subsection 4.B.2). We know that, at first-order, $\delta \mathbf{r}^{\prime}$ is parallel to the plane $\left(P^{\prime}\right)$ normal to $\mathbf{r}^{\prime}$ at $M$; then it suffices to consider the vectorial function $f$ giving the orthogonal projection of $\mathbf{r}^{\prime}$ onto $\left(P^{\prime}\right)$ with the two independent components of $n^{\prime} \mathbf{u}^{\prime}$ on $\left(\pi^{\prime}\right)$, and finally the $2 \times 2$ Jacobian matrix $(\mathbf{C})$ of $f$ at $M$ only, to find a formally simple expression in the first-order approximation:

$$
\delta \mathbf{r}^{\prime} \cong(\mathbf{C}) \mathbf{M Q}
$$

With a local output medium, for example, the refracted ray considered is always normal to the index surface $\left(\Sigma^{\prime}\right)$ at $Q$, then $\left(P^{\prime}\right)$ and $\left(\pi^{\prime}\right)$ coincide and $(\mathbf{C})$ depends on the curvature properties of $\left(\Sigma^{\prime}\right)$ at $M$ [32]. So with Eqs. (4) and (5), we have a generalization of the simple case described by Eq. (3).

\section{Paraxial Image Formation by a Refracting Surface (D) Between an Isotropic and an Anisotropic Medium}

\section{A. Example: Case of a Spherical Refracting Surface With} a Birefringent Uniaxial Medium Whose Optic Axis is Parallel to its Optical Axis

Here I will study the formation of the extraordinary image only (the ordinary being the same as with an isotropic medium of index $n_{0}$ ) by a spherical refracting surface $(D)$, whose optical axis is $\left(\Delta_{g}\right)$, vertex $S$, and center $C$ both on $\left(\Delta_{g}\right)$ (see Fig. 3). Let us note that, for an object point $A$ located on $\left(\Delta_{g}\right)$, the polarization of each extraordinary (ordinary) emerging ray is rectilinear parallel (perpendicular) to its meridian plane.

First, let us determine the paraxial image $A^{\prime}$ of an object point $A$ located on $\left(\Delta_{g}\right)$. At a point $I$ of $(D)$ near $S$, the incidence angle of a ray (or its continuation) coming from $A$ is

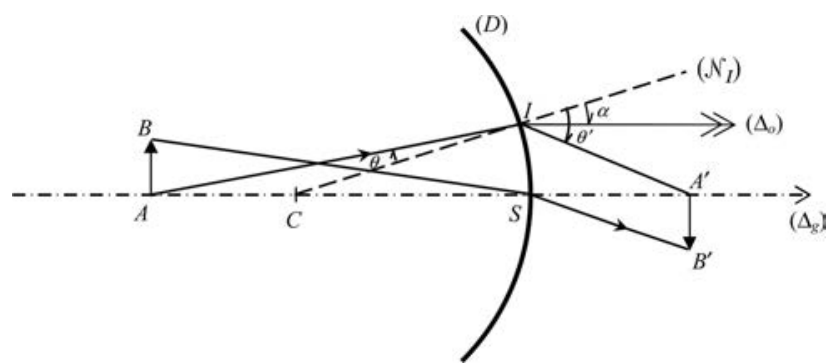

Fig. 3. Imaging of the transverse object $A B$ by a spherical refracting surface $(D)$ with $\left(\Delta_{o}\right)$ parallel to $\left(\Delta_{g}\right)$. As in Fig. 1, the optic axis $\left(\Delta_{o}\right)$ is marked by a double arrow. The positive orientation for the horizontal (vertical) algebraic lengths is to the right (to the top), and for the angles it is counterclockwise. On this particular figure $\overline{A^{\prime} B^{\prime}}, \overline{S C}, \theta^{\prime}$, and $\alpha$ are negative, but the validity of the algebraic relations [Eqs. (6)-(9) and (15)-(24)] is independent of this particular case.

$$
\theta \cong \frac{\overline{S I}}{\overline{A S}}+\frac{\overline{S I}}{\overline{S C}}
$$

The input medium is assumed isotropic, and the output medium uniaxial with $\left(\Delta_{o}\right)$ parallel to $\left(\Delta_{g}\right)$. Hence the inclination of $\left(\Delta_{o}\right)$ with respect to the normal $\left(N_{I}\right)$ to $(D)$ at $I$ is (see Fig. 3):

$$
\alpha \cong \overline{\overline{S I}}
$$

The refracted ray (or its continuation) passes by $A^{\prime}$ on $\left(\Delta_{g}\right)$, because it is in the principal section plane containing $I$ and $\left(\Delta_{g}\right)$; its refraction angle is

$$
\theta^{\prime} \cong \frac{\overline{S I}}{\overline{A^{\prime} S}}+\frac{\overline{S I}}{\overline{S C}}
$$

Placed in Kepler's law; [Eq. (3)], which is valid independently of the sign of $n_{e}-n_{o}$, these expressions [Eqs. (6)-(8)] give the Descartes's position relation with origin at $S$ :

$$
\frac{n_{A}}{\overline{S A^{\prime}}}-\frac{1}{\overline{S A}}=\frac{n_{o}-1}{\overline{S C}} .
$$

Whatever the sign of $n_{A}-n_{o}$, the walk-off makes $n_{A}$ appear instead of $n_{o}$ in the left-hand side of Eq. (9); and in the right-hand side, the presence of $n_{o}$ instead of $n_{A}$ despite the walk-off is due to its variation with the inclination $\alpha$ of the optic axis $\left(\Delta_{o}\right)$ on $\left(\mathcal{N}_{I}\right)$, which depends on the incidence height $\overline{S I}$ according to Eq. (7).

To know whether there is approximate stigmatism for an object point $B$ close to $\left(\Delta_{g}\right)$ in the Gauss conditions (see Fig. 3), I will consider the transfer matrix for the rays crossing the thin system $(D)$; one bit of information given by this matrix is a relation involving the transverse components, i.e., normal to $\left(\Delta_{g}\right), \mathbf{r}_{\perp}$ and $\mathbf{r}_{\perp}{ }^{\prime}$ of the unit vectors $\mathbf{r}$ and $\mathbf{r}^{\prime}$ parallel to the incident and emerging rays.

But first, let us be reminded that on a usual spherical refracting surface, i.e., for its principal planes, the transfer matrix (T) allows to present [33] the fundamental relations between any paraxial ray incident on $I$ and the corresponding ray emerging from (D) under the form:

$$
\left(\begin{array}{l}
\mathbf{S I} \\
\mathbf{r}_{\perp}^{\prime}
\end{array}\right) \cong(\mathbf{T})\left(\begin{array}{l}
\mathbf{S I} \\
\mathbf{r}_{\perp}
\end{array}\right)
$$

with

$$
(\mathbf{T})=\left[\begin{array}{cc}
1 & 0 \\
-P / n & 1 / n
\end{array}\right],
$$

where $n(P)$ denotes the relative refractive index of the output isotropic medium [the power of $(D)$ ] 
with respect to the input isotropic medium. In this case

$$
P=\frac{n-1}{\overline{S C}}
$$

and the Descartes's magnification relation with origin at $S$ involves $n$ : with $B$ and its paraxial image $B^{\prime}$ off the $\left(\Delta_{g}\right)$ axis, $A$ and $A^{\prime}$ their orthogonal projections on $\left(\Delta_{g}\right)$, in the Gauss conditions, for the incident ray $(B I)$ and the associated emerging ray $\left(I B^{\prime}\right)$ we have, using $I=S$ (see Fig. 3) and according to Eq. (10),

$$
\frac{\mathbf{A}^{\prime} \mathbf{B}^{\prime}}{\overline{S A^{\prime}}} \cong \mathbf{r}_{\perp}{ }^{\prime} \cong \frac{1}{n} \mathbf{r}_{\perp} \cong \frac{1}{n} \frac{\mathbf{A B}}{\overline{S A}},
$$

hence (in the limit where $B$ tends to $A$ and $B^{\prime}$ to $A^{\prime}$ ):

$$
\frac{\overline{A^{\prime} B^{\prime}}}{\overline{A B}}=\frac{1}{n} \frac{\overline{S A^{\prime}}}{\overline{S A}} .
$$

For $(D)$ between isotropic and anisotropic media with $\left(\Delta_{o}\right)$ parallel to $\left(\Delta_{g}\right)$, it is clear ([5], Subsection 6.A) that

$$
(\mathbf{T})=\left[\begin{array}{cc}
1 & 0 \\
\frac{1-n_{o}}{n_{A} \overline{S C}} & \frac{1}{n_{A}}
\end{array}\right] .
$$

With a local medium, the existence of such a simple matrix comes from the fact that the point $M$ of the index surface is an umbilic: then $(\mathbf{C})$ is a scalar matrix. Comparing the two transfer matrices (T) above, we deduce that for the extraordinary rays all the paraxial properties of $(D)$, including the existence of the paraxial image $B^{\prime}$ of the object point $B$, are those of a thin system of power $\left(n_{o}-1\right) / \overline{S C}$ with respect to the input isotropic medium, with principal points $H=H^{\prime}=S$, between an input medium of index 1 and an isotropic output medium of index $n_{A}$ (instead of $n_{o}$ ); hence Descartes' formula, with origin at $S$, for the lateral magnification $m_{l}$ is

$$
m_{l}=\frac{\overline{A^{\prime} B^{\prime}}}{\overline{A B}}=\frac{1}{n_{A}} \overline{\overline{S A}},
$$

and the ratio of the focal length of the image space,

$$
f^{\prime}=\overline{S F^{\prime}}=\frac{n_{A}}{n_{o}-1} \overline{S C},
$$

to the focal length of the object space,

$$
f=\overline{S F}=-\frac{1}{n_{o}-1} \overline{S C}
$$

$$
f^{\prime} / f=-n_{A} .
$$

A noticeable difference from the paraxial properties of a usual spherical refracting surface is that its merged first and second nodal points $N=N^{\prime}$, on $\left(\Delta_{g}\right)$, are not the center $C$ of curvature of $(D)$, but such that

$$
\overline{S N}=\frac{n_{A}-1}{n_{o}-1} \overline{S C},
$$

hence the power of $(D)$ may still be written as $\left(n_{A}-1\right) / \overline{S N}$; consequently, $C$ is no more its own image $C^{\prime}$, and

$$
\begin{gathered}
f^{\prime}=\frac{n_{A}-1}{n_{o}-1} \overline{S C}-\frac{-1}{n_{o}-1} \overline{S C}=\overline{H N}-\overline{H F}=\overline{F N} \neq \overline{F C}, \\
f=\frac{n_{A}-1}{n_{o}-1} \overline{S C}-\frac{n_{A}}{n_{o}-1} \overline{S C}=\overline{F^{\prime} N} \neq \overline{F^{\prime} C}, \\
f+f^{\prime}=\overline{S N}=\overline{H N}=\overline{H^{\prime} N^{\prime}} \neq \overline{S C} .
\end{gathered}
$$

\section{Remarks}

- Using the absolute ordinary and extraordinary indices $N_{o}$ and $N_{e}$ of the uniaxial medium, Eq. (9) applied to $A=C$ gives

$$
\overline{S C^{\prime}}=\frac{n_{A}}{n_{o}} \overline{S C}=\left(\frac{N_{e}}{N_{o}}\right)^{2} \overline{S C} .
$$

This result does not depend on the absolute refractive index $N_{i}$ of the isotropic input medium, physically because each incident ray (or its continuation) passing by $C$ is normal to the refracting surface $(D)$.

- The Newton's position and magnification relations can be applied, using the results in Eqs. (16) and (17).

- The Descartes's relations may be written with the origin at $N$ : we find

$$
\frac{1}{\overline{N A^{\prime}}}-\frac{n_{A}}{\overline{N A}}=\frac{1-n_{A}}{\overline{N S}}=\frac{1-n_{o}}{\overline{C S}}, \quad m_{l}=\frac{\overline{N A^{\prime}}}{\overline{N A}} .
$$

- The Lagrange-Smith-Helmholtz relation can be deduced from the determinant of $(\mathbf{T})$, whose value is $1 / n_{A}$, and which is equal to the product of $m_{l}$ by the angular magnification $m_{a}$.

- For a refracting system $\left(D^{\prime}\right)$, which is $(D)$ reversed, the optical reciprocity (i.e., the reversibility of light rays) allows to deduce the corresponding relations in a straightforward permuting $A^{\prime}$ and $A$ (as well as $B^{\prime}$ and $B$ ) in the relations for $(D)$ : 


$$
\begin{aligned}
\frac{1}{\overline{S A^{\prime}}}-\frac{n_{A}}{\overline{S A}}=\frac{1-n_{o}}{\overline{S C}}=\frac{1-n_{A}}{\overline{S N}}, \quad m_{l}=n_{A} \overline{\overline{S A}} \\
\frac{n_{A}}{\overline{N A^{\prime}}}-\frac{1}{\overline{N A}}=\frac{n_{A}-1}{\overline{N S}}=\frac{n_{o}-1}{\overline{C S}}, \quad m_{l}=\frac{\overline{N A^{\prime}}}{\overline{N A}}, \\
m_{l} m_{a}=n_{A} .
\end{aligned}
$$

- With $n_{o}>1,(D)$ and $\left(D^{\prime}\right)$ are convergent systems if $C$ is in the uniaxial medium; in which case, however, the merged nodal points $N$ could be in the isotropic medium if $n_{A}<1$, i.e., $N_{e}^{2} / N_{o}<N_{i}<N_{o}$. The situation occurs only in the negative uniaxial case, easily, for example, at $589 \mathrm{~nm}$ with calcite $\left(N_{o} \cong 1.658, N_{e} \cong 1.486\right)$, and ethanol $\left(N_{i} \cong 1.361\right)$, or glycerin $\left(N_{i} \cong 1.475\right)$, or toluene $\left(N_{i} \cong 1.496\right)$, or an immersion oil for microscopes $\left(N_{i} \cong 1.517\right)$.

- With a positive medium such as sellaite $\left(\mathrm{MgF}_{2}: N_{o} \cong 1.378, N_{e} \cong 1.390\right)$ it is possible to obtain an unusual case analogous to the precedent, where $C$ and $N$ are on both sides of $S$, using heptane $\left(N_{i} \cong 1.388\right)$ for the isotropic medium: here the required conditions are $N_{o}<N_{i}<N_{e}^{2} / N_{o}$.

- If $n_{e}=\sqrt{n_{o}}$ (a fine-tuning could be performed by temperature or wavelength variations, for example), then $n_{A}=1$ and for the paraxial extraordinary rays, the properties of such a single refracting surface, such as $(D)$ or $\left(D^{\prime}\right)$, are those of a usual thin lens: $S=N, f=-f^{\prime}$. This is quite the case with calcite and water.

- For the ordinary paraxial image, $n_{A}$ has to be replaced by $n_{o}$ in Eqs. (9) and (14)-(21), and also $N$ by $C$ in Eqs. (25) and (26), but we see by Eq. (17) that the ordinary and extraordinary focal lengths of the object space are identical. This is not true for the focal lengths of the image space, so the ordinary and extraordinary images of an object are generally different.

\section{B. Case of a Spherical Refracting Surface With a More General Geometry for the Anisotropic Medium}

Here I consider again an arbitrary anisotropic medium-hence possibly uniaxial with $\left(\Delta_{o}\right)$ not parallel to $\left(\Delta_{g}\right)$. For an incident ray carried by $\left(\Delta_{g}\right)$, the unit vector $\mathbf{r}_{N}^{\prime}$ oriented like a refracted ray is not parallel to $\left(\Delta_{g}\right)$ a priori, because of the walk-off. For a paraxial modification of the incident ray, the displacement of the incidence point on $(D)$ from $S$ to $I$ is small compared to $S C$, and the variation $\delta \mathbf{r}$ of $\mathbf{r}$ is nearly equal to its new transverse component $\mathbf{r}_{\perp}$. Transposing the fundamental results of Subsection 2.B, the direction change of the refracted ray considered is given by

$$
\delta \mathbf{r}^{\prime} \cong(\mathbf{C})(\mathbf{P})^{-1}\left[\left(n_{g}-1\right) \delta \mathbf{v}+\mathbf{r}_{\perp}\right], \quad \delta \mathbf{v} \cong-\mathbf{S I} / \overline{S C},
$$

where $n_{g}$ denotes the relative refractive index of the anisotropic medium for the corresponding wave normal parallel to $\left(\Delta_{g}\right)$.
For a paraxial incident pencil of rays from the object point $A$ on $\left(\Delta_{g}\right)$,

$$
\left(n_{g}-1\right) \delta \mathbf{v}+\mathbf{r}_{\perp} \cong\left[-\frac{n_{g}-1}{\overline{S C}}+\frac{1}{\overline{A S}}\right] \mathbf{S I} .
$$

Even in the case when the anisotropic medium is local and when the incident paraxial pencil is conic and rotationally symmetric around $\left(\Delta_{g}\right)$, because of the walk-off the refracted pencil is generally fundamentally astigmatic - and we already know that its corresponding median ray is not on $\left(\Delta_{g}\right)$ but is parallel to $\mathbf{r}_{N}{ }^{\prime}$. Moreover, if no eigenvector of $(\mathbf{C})$ is perpendicular to $\left(\Delta_{g}\right)$ and $\mathbf{r}_{N}{ }^{\prime}$ at the same time, the shape of this refracted pencil is similar to the shape obtained when a conic and rotationally symmetric incident pencil is sent obliquely on an aspheric surface separating two isotropic media, such that at the incidence point of its median ray no principal curvature direction of the surface be parallel to the incidence plane of this ray. But the focal lines of the refracted pencil, when $(\mathbf{C})$ is symmetric, are parallel to the eigenvectors of $(\mathbf{C})$ - and they are mutually orthogonal; their directions do not depend on $\overline{S A}$, contrarily to their distance to $S$. But for nonlocal media the refracted pencil could present a higher-order form of astigmatism ([34], Section 3).

\section{Case of a Plane Refracting Surface}

The case of a plane refracting surface $(D)$ can be easily studied at this stage: one simply has to put $\delta \mathbf{v}=\mathbf{0}$ in Eqs. (28) and (29). The conclusions obtained above about the astigmatism of the paraxial refracted pencil remain valid.

Let us consider the particular case where the anisotropic medium is uniaxial, with a small angle $\alpha$ between the normal to $(D)$ and the direction of $\left(\Delta_{o}\right)$. The angular magnification produced by $(D)$ is, because $(D)$ is plane, the derivative of $\theta^{\prime}$ with respect to $\theta$ : its paraxial value is approximately $1 / n_{A}$ (not $1 / n_{o}$ ) for the extraordinary image (because of the walk-off).

Moreover, if $n_{e}=\sqrt{n_{o}}$, for the extraordinary rays the paraxial properties of this single surface are those of an ordinary wedge (i.e., a prism of small angle), producing in its principal section plane, cf. Eq. (3), the deviation:

$$
\theta^{\prime}-\theta \cong-\left(n_{o}-1\right) \alpha .
$$

Then, if $\alpha=0$, any object for this system merges with its extraordinary paraxial image; but the ordinary paraxial image is different, except for an object located on $(D)$.

\section{Paraxial Properties of Anisotropic Spherical Lenses in Isotropic Media}

A spherical lens is formed by two spherical refracting surfaces $\left(D_{1}\right)$ and $\left(D_{2}\right)$ of optical axis $\left(\Delta_{g}\right)$, vertices $S_{1}$ and $S_{2}$, and centers $C_{1}$ and $C_{2}$ all on $\left(\Delta_{g}\right)$ (see Fig. 4 ). The surrounding media are isotropic, and have the same (relative) refractive index (put equal to 1) for 


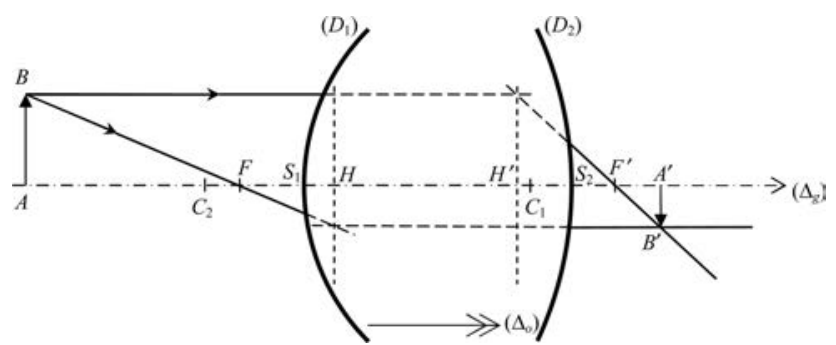

Fig. 4. Transmission through a thick lens formed by two spherical refracting surfaces $\left(D_{1}\right)$ and $\left(D_{2}\right)$ of optical axis $\left(\Delta_{g}\right)$, vertices $S_{1}$ and $S_{2}$ and centers $C_{1}$ and $C_{2}$; and construction of a paraxial image $A^{\prime} B^{\prime}$ using the cardinal points $H, H^{\prime}, F$, and $F^{\prime}$ when $\left(\Delta_{o}\right)$ is parallel to $\left(\Delta_{g}\right)$.

the input of the lens and for its output. $\left(D_{1}\right)$ and $\left(D_{2}\right)$ are crossed by light in this order. The cardinal points of $\left(D_{1}\right)$ or $\left(D_{2}\right)$ will be noted with the subscripts 1 or 2 , respectively.

\section{A. Thin Lenses}

For this subsection, I will assume the thin lens approximation; it permits one to consider that the vertices $S_{1}$ and $S_{2}$ of $\left(D_{1}\right)$ and $\left(D_{2}\right)$ merge in the relations between objects and images, and that the incidence heights on $\left(D_{1}\right)$ and $\left(D_{2}\right)$ of corresponding rays are equal: $S_{1} I_{1} \cong S_{2} I_{2} \equiv S I$. Paraxial properties of thin lenses are entirely determined by the position of one of their focal points $F^{\prime}$ or $F$.

\section{Example: Spherical Lens Made of a Uniaxial} Medium Whose Optic Axis is Parallel to its Optical Axis

Here again, we will study the formation of the extraordinary image only (the ordinary being the same as with an isotropic medium of index $n_{0}$ ). In this case we just have to write, for $\left(D_{1}\right)$ and $\left(D_{2}\right)$, the relations deduced from Subsection 3.A, and to combine them. As a consequence, the terms involving the position of the intermediate image formed by $\left(D_{1}\right)$ cancel (because $S_{1} \cong S_{2} \equiv S$ ); hence we find the classical lens equation, involving its power $P$ (with respect to the input isotropic medium), which is the sum of the powers of $\left(D_{1}\right)$ and $\left(D_{2}\right)$ :

$$
\begin{aligned}
P & =\frac{1}{\overline{S F^{\prime}}}=-\frac{1}{\overline{S F}}=\left(n_{A}-1\right)\left(\frac{1}{\overline{S_{1} N_{1}}}-\frac{1}{\overline{S_{2} N_{2}}}\right) \\
& =\left(n_{o}-1\right)\left(\frac{1}{\overline{S_{1} C_{1}}}-\frac{1}{\overline{S_{2} C_{2}}}\right),
\end{aligned}
$$

[which agrees with Eq. (60) of [5]].

\section{Important Remarks}

- The last expression of $P$ is not evident in terms of rays, but in terms of phase transformation in Gauss conditions: one immediately finds it if we consider that light crossing the thin lens, of local thickness $e$ at the distance $\overline{S I}$ from its optical axis $\left(\Delta_{g}\right)$, undergoes an optical path variation $\left(n_{o}-1\right) e$, which transforms a spherical incident wave into an emerg- ing one [35] because

$$
e \cong \overline{S_{1} S_{2}}-\left(\frac{1}{\overline{S_{1} C_{1}}}-\frac{1}{\overline{S_{2} C_{2}}}\right) \frac{\overline{S I}^{2}}{2}
$$

Note that, because the extraordinary wave normals in Gauss conditions are approximately parallel to the optic axis inside the lens, the refractive index for these waves does indeed appear, rather than $n_{A}$, in the calculation of the phase difference due to the crossing of the lens; and this index is approximately $n_{o}$.

- For the ordinary rays, the power of the lens is also given by the result of Eq. (31). Hence the paraxial properties of the ordinary and extraordinary rays with such a lens are identical; the paraxial ordinary and extraordinary images of any paraxial object merge (and from this point of view the lens is equivalent to a usual, i.e., isotropic, thin lens). But, out of the paraxial domain, the aberrations of these images differ.

\section{Case of a Spherical Lens With a More General} Geometry for the Anisotropic Medium

I consider now a thin lens formed by an arbitrary anisotropic medium (as in Subsection 3.A), and I will prove that, separately for each of the two images of an object, its paraxial properties are also those of a usual thin lens (as in Subsection 4.A.1).

Let us call $n_{g}$ one of its two refractive indices for a wave normal parallel to its optical axis $\left(\Delta_{g}\right)$. Then the argument in the first important remark just above (concerning the transformation of spherical waves) still applies-replacing $n_{o}$ by $n_{g}$-and we find the power of the lens:

$$
P=\frac{1}{\overline{S F^{\prime}}}=-\frac{1}{\overline{S F}}=\left(n_{g}-1\right)\left(\frac{1}{\overline{S C_{1}}}-\frac{1}{\overline{S C_{2}}}\right)
$$

The simplicity of this derivation is astonishing, compared to the complexity of the walk-off phenomena inside the lens and of the Kepler's law that describe them, when the lens is made of a biaxial medium or of fluorite $\left(\mathrm{CaF}_{2}\right.$ : an interesting example of a nonlocal medium; see Subsection 4.B.2). Let us look directly at why these complications concerning the rays do not primarily affect the paraxial pencils emerging from such a thin lens.

For $\left(D_{1}\right)$, crossed by the light propagating from the isotropic input medium to the anisotropic medium, transposing the relation (28) we get

$$
\delta \mathbf{r}^{\prime} \cong(\mathbf{C})(\mathbf{P})^{-1}\left[\left(n_{g}-1\right) \delta \mathbf{v}_{1}+\mathbf{r}_{\perp}\right]
$$

with

$$
\delta \mathbf{v}_{1} \cong-\mathbf{S}_{1} \mathbf{I}_{1} / \overline{S_{1} C_{1}}
$$

where $\mathbf{r}^{\prime}$ is the unit vector oriented like the refracted ray considered inside the lens. But for $\left(D_{2}\right)$, the light propagates from the anisotropic to the isotropic sur- 
rounding medium; hence Eq. (34) must be inverted, $\delta \mathbf{v}_{1}$ must be replaced by

$$
\delta \mathbf{v}_{2} \cong+\mathbf{S}_{2} \mathbf{I}_{2} / \overline{S_{2} C_{2}},
$$

and $\left(\delta \mathbf{r}^{\prime}, \mathbf{r}_{\perp}\right)$ by $\left(-\delta \mathbf{r}^{\prime},-\mathbf{r}_{\perp}{ }^{\prime \prime}\right)$ where $\mathbf{r}_{\perp}$ " is the transverse component of $\mathbf{r}^{\prime \prime}$, the unit vector oriented like the emerging ray considered at the output of the lens, $-\mathbf{r}^{\prime}$ and $-\mathbf{r}^{\prime \prime}$ being turned like $\mathbf{v}_{2}$ (from the isotropic output medium to the anisotropic medium),

$$
-\mathbf{r}_{\perp}{ }^{\prime} \cong(\mathbf{P})(\mathbf{C})^{-1}\left(-\delta \mathbf{r}^{\prime}\right)-\left(n_{g}-1\right) \delta \mathbf{v}_{2},
$$

hence

$$
\mathbf{r}_{\perp}{ }^{\prime} \cong\left(n_{g}-1\right)\left(\delta \mathbf{v}_{1}+\delta \mathbf{v}_{2}\right)+\mathbf{r}_{\perp} .
$$

Because the thickness of the lens is neglected for the ray tracing, the effect of the transversal shift of the refracted ray inside the lens vanishes, even in the presence of walk-off,

$$
\mathbf{S}_{1} \mathbf{I}_{1} \cong \mathbf{S}_{2} \mathbf{I}_{2} \equiv \mathbf{S I}
$$

(for a calcite lens the maximum value of the transverse component of $\mathbf{I}_{1} \mathbf{I}_{2}$ is near $0.5 \mathrm{~mm}$ only for a local thickness of $5 \mathrm{~mm}$, and is reached when $\alpha \cong 42^{\circ}$; see, for example, [31] and [27], pp. 541-542 and 546); but this approximation is still better when there is a weaker walk-off, as in Subsection 4.A.1. For such a thin lens, between its principal planes, its transfer matrix is deduced from Eqs. (34)-(38),

$$
(\mathbf{T})=\left[\begin{array}{cc}
1 & 0 \\
-P & 1
\end{array}\right],
$$

and involves the power of the lens given above [Eq. 33] for one of the two transmitted waves.

The matrix (C) has disappeared in Eq. (37), hence also in the expression of (T) - for the transmitted wave considered-and in the paraxial properties of the thin lens. Moreover, the fundamental (i.e., firstorder) astigmatisms due to the two surfaces of the thin lens compensate each other. For the transmitted wave considered, this thin lens has therefore the same paraxial properties as a usual thin lens; but an important difference with Subsection 4.A. 1 is that the two transfer matrices for the two transmitted waves are generally not identical, therefore such a lens does give two paraxial images of an object.

\section{Remark}

If the anisotropic medium is uniaxial with $\left(\Delta_{o}\right)$ not parallel to $\left(\Delta_{g}\right)$, the extraordinary (ordinary) image is very bright with respect to the other if the paraxial incident beam is polarized linearly in a direction approximately parallel (perpendicular) to the plane containing $\left(\Delta_{o}\right)$ and $\left(\Delta_{g}\right)$ : this is probably the only relevant reason to distinguish two cases when $\left(\Delta_{o}\right)$ is orthogonal to $\left(\Delta_{g}\right)$; compare to the cases B and $\mathrm{C}$ of
Sections 6 and 7 of [5]. In [5], we can see that the expression (61) of the lens power of [5]-deduced from its Eqs. (20)-(25) or (42)-disagrees with my expression (33) derived in this subsection. The origin of this discrepancy may lie in Eqs. (20) and (23) of [5], whose derivation is not detailed.

\section{B. Thick Lenses}

The simplicity of the derivation of the wavefront transformation by thin lenses, due to the fact that $\mathbf{S}_{1} \mathbf{I}_{1} \cong \mathbf{S}_{2} \mathbf{I}_{2}$, disappears for thick lenses. This is already true, even in Gauss conditions, with lenses made of isotropic media, when the difference between $\mathbf{S}_{1} \mathbf{I}_{1}$ and $\mathbf{S}_{2} \mathbf{I}_{2}$ cannot be neglected in view of the lens thickness and the ray obliquity. With thick lenses made of an anisotropic medium, the walk-off phenomenon changes that obliquity and further complicates the expression of $\mathbf{S}_{2} \mathbf{I}_{2}-\mathbf{S}_{1} \mathbf{I}_{1}$; but for several types of birefringent thick lenses, we will be able to easily derive the consequences of the walk-off on the paraxial properties.

\section{First Example: Case of a Lens Made of a}

Uniaxial Medium Whose Optic Axis is Parallel to its Optical Axis

To start with, at that stage it is easy to study the paraxial properties of a thick lens made of a birefringent uniaxial medium whose optic axis is parallel to its optical axis $\left(\Delta_{g}\right)$ (see Fig. 4). The rotational symmetry around $\left(\Delta_{g}\right)$ leads, for the ordinary and the extraordinary images separately, to approximate stigmatism near $\left(\Delta_{g}\right)$ : there is no first-order astigmatism. For the same reason as in Subsection 4.A.1, I will study the formation of the extraordinary image only. Here again, the paraxial influence of the walkoff is entirely summarized in Descartes's relations by the replacement of $n_{o}$ by $n_{A}$, and of a curvature center by a nodal point, for each surface of the lens (see Subsection 3.A). Combining these relations for $\left(D_{1}\right)$ and $\left(D_{2}\right)$, and introducing $\overline{S_{1} S_{2}}$, which is not neglected, we find that the paraxial properties of such a thick lens are yet entirely characterized by the position of a few cardinal points on $\left(\Delta_{g}\right)$ : the first and second principal points $H$ and $H^{\prime}$, and the focal points $F$ and $F^{\prime}$, such that $f=\overline{H F}$ and $f^{\prime}=\overline{H^{\prime} F^{\prime}}$. They are given by classical formulas [36], which are adapted here replacing $n$ (the index of an isotropic lens) by $n_{A}$, and the curvature center $C_{1}$ of $\left(D_{1}\right)$ by its nodal point $N_{1}$; idem for $\left(D_{2}\right)$. We obtain

$$
\begin{aligned}
\frac{1}{f^{\prime}} & =\left(n_{A}-1\right)\left(\frac{1}{\overline{S_{1} N_{1}}}-\frac{1}{\overline{S_{2} N_{2}}}+\frac{n_{A}-1}{n_{A}} \frac{\overline{S_{1} S_{2}}}{\overline{S_{1} N_{1} \overline{S_{2} N_{2}}}}\right) \\
& =\frac{n_{A}-1}{n_{A}} \frac{n_{A} \overline{N_{1} N_{2}}-\overline{S_{1} S_{2}}}{\overline{S_{1} N_{1}} \overline{S_{2} N_{2}}} \\
& =\left(n_{o}-1\right)\left(\frac{1}{\overline{S_{1} C_{1}}}-\frac{1}{\overline{S_{2} C_{2}}}+\frac{n_{o}-1}{n_{A}} \frac{\overline{S_{1} S_{2}}}{\overline{S_{1} C_{1}} \overline{S_{2} C_{2}}}\right)=-\frac{1}{f},
\end{aligned}
$$




$$
\frac{\overline{H H^{\prime}}}{f^{\prime}}=\frac{\left(n_{A}-1\right)^{2}}{n_{A}} \frac{\overline{S_{1} S_{2}} \overline{N_{1} N_{2}}}{\overline{S_{1} N_{1}} \overline{S_{2} N_{2}}}=\frac{\left(n_{o}-1\right)^{2}}{n_{A}} \frac{\overline{S_{1} S_{2}} \overline{N_{1} N_{2}}}{\overline{S_{1} C_{1}} \overline{S_{2} C_{2}}},
$$

$$
\begin{gathered}
\frac{\overline{S_{1} H}}{f^{\prime}}=-\frac{n_{A}-1}{n_{A}} \frac{\overline{S_{1} S_{2}}}{\overline{S_{2} N_{2}}}=-\frac{n_{o}-1}{n_{A}} \frac{\overline{S_{1} S_{2}}}{\overline{S_{2} C_{2}}}, \\
\frac{\overline{S_{2} H^{\prime}}}{f^{\prime}}=-\frac{n_{A}-1}{n_{A}} \frac{\overline{S_{1} S_{2}}}{\overline{S_{1} N_{1}}}=-\frac{n_{o}-1}{n_{A}} \frac{\overline{S_{1} S_{2}}}{\overline{S_{1} C_{1}}},
\end{gathered}
$$

(these results agree with Eqs. (39)-(41) of [5]). We find also the Lagrange-Smith-Helmholtz relation, by multiplication of the corresponding relations for $\left(D_{1}\right)$ and $\left(D_{2}\right)$,

$$
m_{l} m_{a}=\left(1 / n_{A}\right) n_{A}=1,
$$

hence the first and second nodal points $N$ and $N^{\prime}$ of the thick lens are at $H$ and $H^{\prime}$, respectively.

Knowing the position of these cardinal points for the extraordinary beams, it becomes possible to use all the classical relations between objects and images. For the ordinary paraxial beams, the cardinal points are given by equations analogous to Eqs. (40)-(43), but where $n_{A}$ has to be replaced by $n_{o}, N_{1}$ by $C_{1}$, and $N_{2}$ by $C_{2}$, we see that the distances between the ordinary and extraordinary corresponding cardinal elements are of first order in $\overline{S_{1} S_{2}}$, hence are negligible if $\overline{S_{1} S_{2}}$ is negligible with respect to $\overline{S_{1} C_{1}}$ and $\overline{S_{2} C_{2}}$ (thin lens approximation).

\section{Second Example: Case of a Fluorite Lens Whose Optical Axis has the Direction [1 11 1]}

Recently, an unusual form of birefringence has been observed in fluorite and crystals of the same class, due to nonlocality (i.e., spatial dispersion) [37,38]. The semiconductor industry may some day use fluorite lenses in the photolithography objectives, which work in vacuum ultraviolet, because fluorite is the best material among the few transparent media in this spectral range. To reduce the aberrations due to this birefringence, which cannot be neglected when using the excimer laser line at $157 \mathrm{~nm}$, it has been proposed to combine lenses whose optical axes $\left(\Delta_{g}\right)$ are parallel to the $\left[\begin{array}{lll}1 & 1 & 1\end{array}\right]$ direction of the conventional cubic cell $(C)$ of this class of crystals.

For such media, we actually know that the walk-off is more difficult to study than with conventional birefringent crystals, because the rays are no more perpendicular to the index surface $(\Sigma)$. This authorizes the absence, recently reported [28], of internal conical refraction associated to the conical points of $(\Sigma)$, all located on the four optic axes parallel to the diagonals of $(C)$. We must note that there are three additional optic axes for these crystals, parallel to the edges of (C). I have recently shown [34] that when the angle between a wave vector and an optic axis is of first order, the walk-off angle $\chi$ is of first order near the latter optic axes, and, surprisingly, of second order near the former.

This property allows to consider other parameters (the thicknesses of the lenses) to reduce the aberrations due to the birefringence of thick fluorite lenses in objectives having $\left(\Delta_{g}\right)$ parallel to the $\left[\begin{array}{lll}1 & 1 & 1\end{array}\right]$ direction. But in order to use such lenses the first question is "What are their paraxial properties?" The answer is very simple. Indeed, because the walk-off is of second order here, it does not influence the paraxial properties. Moreover, the refractive index variation as a function of the propagation direction is of first order in Gauss conditions, so its effect on the direction of the emerging rays is of second order, and does not affect the paraxial properties; finally, in this situation, the double refraction is also negligible in Gauss conditions, because $\left(\Delta_{g}\right)$ is parallel to an optic axis. Hence the paraxial properties of that thick lens are identical to the properties of a usual one, of refractive index $n_{[111]}$, and there is no fundamental astigmatism.

But when $\left(\Delta_{g}\right)$ is parallel to an optic axis of direction $\left[\begin{array}{lll}1 & 0 & 0\end{array}\right]$, for the paraxial rays the effect of $\chi$ on $\mathbf{S}_{2} \mathbf{I}_{2}-\mathbf{S}_{1} \mathbf{I}_{1}$ cannot be neglected because it is of first order and the lens is thick. Moreover, even for an object point on $\left(\Delta_{g}\right)$, there is no approximate stigmatism because the azimuthal dependence of $\chi$ produces a higher order form of astigmatism (see [34], Section 3).

\section{More General Case}

In a more general case than in Subsections 4.B.1 and 4.B.2, we know that even in Gauss conditions a fundamental astigmatism appears with each refraction on a surface, because of the walk-off (Subsection 3.B). Then, $\mathbf{S}_{2} \mathbf{I}_{2}-\mathbf{S}_{1} \mathbf{I}_{1}$ being not negligible in a thick lens, there is a priori no more systematic compensation of the astigmatisms due to the refractions on its two surfaces (as in Subsection 4.A.2); it has been theoretically predicted [3] and experimentally verified (see [4] and its Fig. 5) that the global first-order astigmatism of a uniaxial thick lens, for the extraordinary image, increases in proportion to $\overline{S_{1} S_{2}}$. Moreover, proportionally to this thickness of the lens also, for an incident ray carried by $\left(\Delta_{g}\right)$, the walk-off generally produces a sensible transversal shift of the emerging ray. In addition, for one incident beam, the double refraction gives two emerging beams. And if the thick lens was biaxial, with one optic axis near $\left(\Delta_{g}\right)$, the internal conical refraction associated to this axis would produce a nonconventional aberration in proportion to $\overline{S_{1} S_{2}}$.

\section{Limit Case: A Slab}

The case of a slab can be considered as the limit of a thick lens with vanishing curvatures of its surfaces. For a slab made of a uniaxial medium of thickness $e$, I consider again the extraordinary rays only; if the optic axis is not perpendicular to the slab, there is a fundamental astigmatism proportional to $e$ [39].

When the curvatures of the parallel surfaces of the slab are null, and the optic axis $\left(\Delta_{o}\right)$ is parallel to the 
normal $(\mathcal{N})$ to the slab, the relations between objects and images for the two surfaces are easy to combine; the result is that the paraxial image is shifted longitudinally, with respect to the object, by a length,

$$
\overline{A A^{\prime}}=e\left(1-\frac{1}{n_{A}}\right),
$$

parallel to $(\mathcal{N})$ and in the sense of the propagation of light. Once more, the walk-off makes that $n_{A}$ appears instead of $n_{o}$ in Eq. (45); hence the position of the paraxial ordinary image differs from the position of the extraordinary. In addition, their spherical aberrations are different. Moreover, if $n_{e}=\sqrt{n_{o}}$, any object for this slab coincides with its extraordinary paraxial image; but the algebraic distance between the ordinary and the extraordinary images of an object point is $e\left(n_{o}{ }^{-1}-n_{A}{ }^{-1}\right)$.

When the angle $\alpha$ between $(\mathcal{N})$ and $\left(\Delta_{o}\right)$ is small, the approximate stigmatism is preserved. But [according to the walk-off angle given by Eq. (3) for $\theta=0$ ] the position of the extraordinary image is classically shifted transversally also, in a direction of the principal section plane parallel to $\left(\Delta_{o}\right)$ and $(\mathcal{N})$, by length $e\left[1-\left(n_{o} / n_{A}\right)\right] \alpha$ independent of the surrounding medium. And if $n_{e}=\sqrt{n_{o}}$, the longitudinal shift vanishes, the paraxial imaging is the same with a pair of identical usual wedges placed head to foot and distant of $e$.

With slabs made of an anisotropic medium with no optic axis quasi-parallel to $(\mathcal{N})$, a fundamental astigmatism appears because of the walk-off. This fact has been well-known for a long time, because it limits the performances of the interferential microscopy system of Lebedeff [40].

\section{Conclusion}

Because the refracted rays are directly given by Huygens's construction, the concept of walk-off is not essential when investigating refraction; but it is interesting when the distinction between rays and wave normals is relevant. This is the case for a clear understanding of the paraxial properties of thin spherical lenses made of an arbitrary anisotropic medium, even with fluorite for which some usual birefringence properties are no more valid.

That finding has encouraged us to scan the relations between objects and images, and to interpret the curvature radius of the umbilic of the extraordinary sheet $\left(\Sigma_{e}\right)$ of a uniaxial index surface as an apparent index $n_{A}$, in order to express these relations easily-when the angle between $\left(\Delta_{o}\right)$ and $\left(\Delta_{g}\right)$ is small-and to point out the fact that for a spherical refracting surface (with a uniaxial medium) its center is no more its nodal point even if $\left(\Delta_{o}\right)$ is parallel to $\left(\Delta_{g}\right)$. Moreover, and again if $\left(\Delta_{o}\right)$ is parallel to $\left(\Delta_{g}\right)$, the ordinary and extraordinary images given by a slab are different, because of the walk-off. We have also seen that, because of the walk-off, a fundamental astigmatism may appear for a refracted pencil, which may limit the interest of thick birefringent systems; but in a thin lens the fundamental astigmatisms associated to its two surfaces compensate each other.

The author thanks Pierre Chavel for his comments.

\section{References and Notes}

1. M. C. Simon, "Ray tracing formulas for monoaxial optical components," Appl. Opt. 22, 354-360 (1983).

2. M. C. Simon and R. M. Echarri, "Ray tracing formulas for monoaxial optical components: vectorial formulation," Appl. Opt. 25, 1935-1939 (1986).

3. H. Shimomura, H. Kikuta, and K. Iwata, "First-order aberration of a double focus lens made of a uniaxial crystal," J. Opt. Soc. Am. A 9, 814-819 (1992).

4. J. P. Lesso, A. J. Duncan, W. Sibbett, and M. J. Padgett, "Aberrations introduced by a lens made from a birefringent material," Appl. Opt. 39, 592-598 (2000).

5. M. Avendaño-Alejo and M. Rosete-Aguilar, "Paraxial theory for birefringent lenses," J. Opt. Soc. Am. A 22, 881-891 (2005).

6. M. J. Downs, W. H. McGivern, and H. J. Ferguson, "Optical system for measuring the profiles of super smooth surfaces," Precis. Eng. 7, 211-215 (1985).

7. C. Chou, J. Shyu, Y. Huang, and C. Yuan, "Common-path optical heterodyne profilometer: a configuration," Appl. Opt. 37, 4137-4142 (1998).

8. K. Kinnstatter, M. Ojima, and S. Yonezawa, "Amplitude detection for focus error in optical disks using a birefringent lens," Appl. Opt. 29, 4408-4413 (1990).

9. J. A. Ghosh and A. K. Chakraborty, "High frequency enhancement using a birefringent lens," Opt. Commun. 40, 329-331 (1982).

10. W. Fiala, "Multifocal intraocular lenses fabricated from media exhibiting tuned birefringence," Optom. Vision Sci. 69, 329332 (1992).

11. O. N. Stavroudis, "Ray tracing formulas for uniaxial crystals," J. Opt. Soc. Am. 52, 187-191 (1962).

12. Q.-T. Liang, "Simple ray tracing formulas for uniaxial optical crystals," Appl. Opt. 29, 1008-1010 (1990).

13. J. Lekner, "Reflection and refraction by uniaxial crystals," J. Phys. Condens. Matter 3, 6122-6133 (1991).

14. W. Q. Zhang, "General ray-tracing formulas for crystals," Appl. Opt. 31, 7328-7331 (1992).

15. Z. Shao and C. Yi, "Behavior of extraordinary rays in uniaxial crystals," Appl. Opt. 33, 1209-1212 (1994).

16. Z. Shao, "Refractive indices for extraordinary waves in uniaxial crystals," Phys. Rev. E 52, 1043-1048 (1995).

17. E. Cojocaru, "Direction cosines and vectorial relations for extraordinary-wave propagation in uniaxial media," Appl. Opt. 36, 302-306 (1997).

18. E. Cojocaru, "Explicit relations for the extraordinary-ray trajectory at the back of a rotating uniaxial birefringent plate," Appl. Opt. 36, 8886-8888 (1997).

19. G. Beyerle and I. S. McDermid, "Ray-tracing formulas for refraction and internal reflection in uniaxial crystal," Appl. Opt. 37, 7947-7953 (1998).

20. E. Cojocaru, "Characteristics of ray traces at the back of biaxial crystals at normal incidence," Appl. Opt. 38, 4004-4010 (1999).

21. M. Avendaño-Alejo, O. Stavroudis, and A. R. Boyain, "Huygens' principle and rays in uniaxial anisotropic media I. Crystal axis normal to refracting surface," J. Opt. Soc. Am. A 19, 1668-1673 (2002).

22. M. Avendaño-Alejo and O. Stavroudis, "Huygens' principle and rays in uniaxial anisotropic media II. Crystal axis with arbitrary orientation,” J. Opt. Soc. Am. A 19, 1674-1679 (2002).

23. M. C. Simon and K. V. Gottschalk, "Optical path in birefringent media and Fermat's principle," Pure Appl. Opt. 7, 14031410 (1998). 
24. M. Avendaño-Alejo and M. Rosete-Aguilar, "Optical path difference in a plane-parallel uniaxial plate," J. Opt. Soc. Am. A 23, 926-932 (2006).

25. G. Chartier, Manuel d'Optique (Hermès, 1997), pp. 240-244.

26. S. Huard, Polarisation de la Lumière (Masson, 1994), pp. $76-84$.

27. G. Bruhat, Cours de Physique Générale-Optique (Masson, 1992).

28. L. Dettwiller, "Absence of internal conical refraction with the spatially dispersive index surface of fluorine; discussion of the orthogonality of the Poynting vector to the index surface," Opt. Express 14, 3339-3344 (2006).

29. But any refracting surface $(D)$ intersecting $\left(\Delta_{g}\right)$ at an umbilic $S$-called the "vertex" of $(D)$-and having at $S$ an osculating sphere of center $C$ on $\left(\Delta_{g}\right)$ also, has the same paraxial optical properties as a spherical refracting surface of center $C$ and vertex $S$.

30. In parametric form and with an $x$ axis parallel to $\left(\Delta_{o}\right)$, the equation of the ellipse (centered at the origin $O$ ) that is the section of $\left(\Sigma_{e}\right)$ by the incidence plane (Fig. 1) is

$$
x=n_{o} \cos t, \quad y=n_{e} \sin t,
$$

hence the radius of curvature is

$$
R=\frac{\left(\dot{x}^{2}+\dot{y}^{2}\right)^{3 / 2}}{\dot{x} \ddot{y}-\ddot{x} \dot{y}}=\frac{\left(n_{o}{ }^{2} \sin ^{2} t+n_{e}{ }^{2} \cos ^{2} t\right)^{3 / 2}}{n_{o} n_{e}},
$$

see, for example, E. W. Weisstein, CRC Concise Encyclopedia of Mathematics, 2nd ed. (Chapman \& Hall/CRC, 2003), p. 869. Because $U$ is an umbilic, $n_{A}$ is this radius $R$ at the point $U$; it corresponds to $t=0$, whence $n_{A}=n_{e}^{2} / n_{o}$.
31. M. Avendaño-Alejo, "Analysis of the refraction of the extraordinary ray in a plane-parallel uniaxial plate with an arbitrary orientation of the optical axis," Opt. Express 13, 2549-2555 (2005).

32. If $M$ is a regular point of $\left(\Sigma^{\prime}\right)$ of class $\mathrm{C}^{2}$, the matrix $(\mathbf{C})$ is symmetric. Moreover, if $M$ is in a symmetry plane $\left(\pi_{S}\right)$ of $\left(\Sigma^{\prime}\right)$, and if $(\mathbf{C})$ is expressed in a basis $\left(\mathbf{u}_{x}, \mathbf{u}_{y}\right)$ where $\mathbf{u}_{x}$ is parallel to $\left(\pi_{S}\right)$ and $\mathbf{u}_{y}$ normal to it, then $(\mathbf{C})$ is diagonal.

33. See, for example, M. V. Klein, Optics (Wiley, 1970), pp. 84105.

34. L. Dettwiller, "Walk-off near optic axes of spatially dispersive media of fluorite symmetry: the case of conical points," J. Mod. Opt. 54, 1173-1185 (2007).

35. J. W. Goodman, Introduction à l'Optique de Fourier et à l'Holographie (Masson, 1972), pp. 73-78.

36. See, for example, L. Dettwiller, Les Instruments d'OptiqueÉtude Théorique, Expérimentale et Pratique, 2nd ed. (Ellipses, 2002), p. 42.

37. J. H. Burnett, Z. H. Levine, and E. L. Shirley, "Intrinsic birefringence in calcium fluoride and barium fluoride," Phys. Rev. B 64, 241102(R) (2001).

38. L. Dettwiller, "Observation récente d'une forme de biréfringence dans certains cristaux à symétrie cubique-théorie et conséquences pratiques," Bull. Un. Prof. Phys. Chim. 99(2), 77-103 (2005).

39. M. C. Simon, "Image formation through monoaxial planeparallel plates," Appl. Opt. 27, 4176-4182 (1988).

40. A. A. Lebedeff, "L'interféromètre à polarisation et ses applications," Rev. d'Opt. 9, 385-413 (1930). 